10 years ESJ

Special edition

\title{
Caracterización de la Intención Emprendedora en Proyectos Universitarios con Base a un Evento Académico en Tiempos de COVID-19
}

\author{
Genesis Hernández Camacho \\ José Luis Favela Rodríguez. \\ Cristian Antonio Bustamante Arneros \\ Sandra Verónica Ramos de la Rosa \\ Gabriela Velasco Rodríguez \\ Universidad Autónoma de Ciudad Juárez, Mexico
}

Doi: $10.19044 /$ esj.2021.v17n4p130

Submitted: 28 October 2020

Accepted: 23 November 2020

Published: 07 February 2021
Copyright 2021 Author(s)

Under Creative Commons BY-NC-ND

4.0 OPEN ACCESS

Cite As:

Camacho H.G., Rodríguez Favela L.J., Bustamante Arneros C.A., Ramos de la Rosa S.V. \& Velasco Rodríguez G. (2021). Caracterización de la Intención Emprendedora en Proyectos Universitarios con Base a un Evento Académico en Tiempos de COVID-19. European Scientific Journal, ESJ, 17(4), 130. https://doi.org/10.19044/esj.2021.v17n4p130

\section{Resumen}

En la presente investigación se retoma a la iniciativa emprendedora en proyectos universitarios que "representa un indicador de esfuerzo individual o grupal y de la disposición a ejecutar un comportamiento cuando sea el momento y la oportunidad adecuada" (Krauss Delorme, Bonomo Odizzio, \& Volfovicz Leon, 2018, pág. 84) ante tiempos de COVID-19. El objetivo es distinguir las características de la intención emprendedora en proyectos de universitarios con base a un evento académico. La metodología aplicada consiste en un análisis de 83 proyectos a través una encuesta digital en el evento Emprendízate 2020-1. Con este insumo se plantea los resultados de tres indicadores correspondientes al perfil del emprendedor, sector y giro económico, así como aquellos factores que contribuyan a la intención emprendedora del proyecto, donde se pone de manifiesto que la interacción con otras personas e internet son la fuente de inspiración. En cambio, los inconvenientes están asociados a la disponibilidad de financiamiento, contar con capacitación y de recursos humanos idóneos para la iniciación de los 
proyectos universitarios.

Palabras clave: Intención emprendedora, proyecto universitario, encuesta, evento académico

\title{
Characterization of Entrepreneurial Intention in University Projects Based on an Academic Event in Times of COVID-19
}

\author{
Genesis Hernández Camacho \\ José Luis Favela Rodríguez. \\ Cristian Antonio Bustamante Arneros \\ Sandra Verónica Ramos de la Rosa \\ Gabriela Velasco Rodríguez. \\ Universidad Autónoma de Ciudad Juárez, Mexico
}

\begin{abstract}
This research takes up the entrepreneurial initiative in university projects that "represents an indicator of individual or group effort and the willingness to execute a behavior when the time and opportunity are right" in times of COVID-19. This paper focuses on distinguishing the characteristics of the entrepreneurial intention in university projects based on an academic event. The applied methodology consists of an analysis of 83 projects through a digital survey at the Emprendízate 2020-2021 event. With this input, the results of three indicators corresponding to the profile of the entrepreneur, sector, and economic line are presented. This was in addition those factors that contribute to the entrepreneurial intention of the project. Here it is shown that interaction with other people and the internet are the major source of inspiration. Instead, the drawbacks are associated with the availability of financing and having training and suitable human resources for the initiation of university projects.
\end{abstract}

Keywords: Entrepreneurial intention, university project, survey, academic event

\section{Introduction}

En la actualidad existe un creciente interés hacia el estudio de las intenciones emprendedoras de los estudiantes universitarios (Rueda Sampero, Fernández-Laviada, \& Herrero Crespo, 2013, pág. 142), donde "La teoría más utilizada y reconocida en la explicación de la formación de intenciones para la creación de nuevos negocios es la Teoría del Comportamiento Planificado (TCP), de Ajzen (1991)" (Echeverri-Sánchez, Valencia-Arias, Benjumea- 
Arias, \& Barrera-Del Toro, 2018, pág. 5). Para ello, es importante considerar la definición de la intención emprendedora antes de seguir:

La intención emprendedora puede ser definida como el estado de la mente en el que la atención de una persona se centra en el cumplimiento de un objetivo, en este caso la creación de una empresa o negocio lo cual tiene cierta influencia en las acciones a tomar por el individuo para llegar a dicho objetivo (Prodan \& Drnovsek, 2010). Respecto a esto, los investigadores han propuesto diversos modelos de intención emprendedora en los que se busca identificar sus factores determinantes como la autoeficacia, factores personales, contextuales, entre otros (Kuttim et al., 2014). Dos modelos han recibido principal atención en la literatura: La teoría del Comportamiento Planeado de Ajzen y el Modelo del Evento Empresarial de Shapero (Karali, 2013, citado por Kuttim et al., 2014) (citado en Valencia Arias, Montoya Restrepo, \& Montoya Restrepo, 2016, págs. 883-884)

Con base a la definición expuesta, se retoman algunos estudios internacionales y nacionales sobre la intención emprendedora. Uno de los estudios que se presentan es aquel que explora la intención emprendedora y los factores que inciden en la misma, para estudiantes universitarios de seis países latinoamericanos provenientes de empresas familiares con una muestra constituida por 771 estudiantes de 8 universidades. El principal aporte de esta investigación es el planteamiento de una ecuación matemática que permita predecir la intención emprendedora mediante cuatro factores: relacionamiento con la empresa, innovación, motivación para emprender y actitudes personales (poder hedonismo), (Krauss Delorme, Bonomo Odizzio, \& Volfovicz Leon, 2018, págs. 86-89).

Otro estudio realizado en Medellín, Colombia, con la aplicación 29 entrevistas semiestructuradas a estudiantes de pregrado del Instituto Tecnológico Metropolitano (ITM), que entre los resultados obtiene que el estudiantado busca crear empresa por la expectativa de independencia laboral consideran ciertas categorías que son: oportunidades para nuevos productos o servicios; influencia familiar o social para crear un negocio; equilibrio entre trabajo y vida privada; situación motivadora. (Echeverri-Sánchez, ValenciaArias, Benjumea-Arias, \& Barrera-Del Toro, 2018, págs. 2,9-15).

Además, existe un trabajo que analiza el efecto de las ventajas e inconvenientes percibidos emprendimiento sobre la intención de crear un negocio propio, tomando como referencia una muestra compuesta por 274 estudiantes de la Facultad de Ciencias Económicas y Empresariales de la Universidad de Cantabria, Santander en España. 
Aporta dos contribuciones respecto a la literatura previa: 1) examina la influencia de las ventajas e inconvenientes que los individuos asocian a la creación de una empresa propia en la intención de emprendimiento, incorporando dichas variables en un modelo de comportamiento global, y 2) investiga el comportamiento emprendedor de los estudiantes universitarios, un colectivo de especial relevancia en este ámbito, dado que se encuentra en una fase previa a la incorporación al mercado laboral, y en la que debe decidir si optara por crear su propia empresa o por trabajar por cuenta ajena (Rueda Sampero, Fernández-Laviada, \& Herrero Crespo, 2013, págs. 151-152).

También, la Facultad de Comercio y Administración de Tampico, Universidad Autónoma de Tamaulipas realizo un estudio del comportamiento emprendedor teniendo en cuenta dos grupos de emprendedores, donde fue posible definir cada uno de ellos con base en las características sociodemográficas (género) y conductuales de sus miembros: 1) Desarrollo Personal y Organizacional, 2) Creatividad, 3) Propensión al riesgo, 4) Autodeterminación, 5) Efectividad en el manejo del cambio y 6) Honestidad) (Ochoa Hernández, Azueta Flores, \& Rangel Lyne, 2015, págs. 30-31).

Por otro lado, hay trabajos referidos a las competencias. (Casimiro, 2019); a la cultura emprendedora (Borrayo Rodríguez, Valdez Zepeda, \& Delgado Melgarejo, 2019); formación del emprendedor (Jolonch \& Ferreira, 2016) (Sánchez-García \& Hernández-Sánchez, 2016); otros modelos y metodologías (Lanero, Vázquez, \& Muñoz-Adáñez, 2015) (Lanero, Vázquez, \& Muñoz-Adáñez, 2015) y análisis bibliométrico sobre intención emprendedora (Valencia Arias, Montoya Restrepo, \& Montoya Restrepo, 2016).

En el estudio formado por Casimiro (2019) busca determinar el nivel de desarrollo de las competencias de emprendimiento empresarial en estudiantes universitarios. Se hizo un estudio descriptivo con diseño transeccional en donde la muestra fue de 212 estudiantes de la carrera profesional de Administración, Finanzas y Negocios Globales de la Universidad Privada TELESUP de Perú, en el año 2018. Se utilizó la encuesta como técnica y como instrumento un cuestionario, el cual estuvo conformado por 20 ítems, cada uno con las siguientes alternativas de respuesta: adecuado, medio o regular o inadecuado.

Otra investigación realizada en Colombia plantea 25 ideas de negocio. Las principales variables que se trabajaron dentro la investigación fueron el conocer de donde surgen las ideas de negocio analizadas, determinar si su ventana de oportunidad en el mercado está abierta o cerrada, así como también 
identificar el perfil de los emprendedores y los mentores para poder determinar que implicación que tuvo en el desarrollo de los emprendimientos de este estudio. El enfoque utilizado es de origen descriptivo cuantitativo, cabe resaltar que los resultados obtenidos presentan la información de que un 86\% de las ideas de negocio nacen de una idea de mercado y no de una innovación tecnológica (Jolonch \& Ferreira, 2016).

Revisando estudios elaborados dentro del país de México, hay investigaciones realizada en la Universidad de Guadalajara, México acerca de la cultura emprendedora en jóvenes universitarios. El objetivo de identificar dicho perfil en los jóvenes para lograr de esta manera promover un modelo universitario orientado a la formación de emprendedores. Dentro de la investigación se realizó un método cuantitativo comparativo, en donde se obtuvieron resultados donde describen que, al inicio de la gestación del emprendimiento, los estudiantes tienden a ser más proactivos en sus aptitudes, aunque tengan limitaciones en los recursos y poco dominio temático de lo que es el emprendimiento, sin embargo, con el transcurso de los primeros dos años muestran desmotivación hacia la actitud emprendedora (Borrayo \& Otros, 2019).

Una vez revisados los anteriores trabajos, se infiere que la intención emprendedora puede ser estudiada desde diferentes perspectivas, ya sean cuantitativa o cualitativa. Otro aspecto son los factores y/o las categorías que deducen o infieren en cada uno de dichos estudios.

Ahora bien, en la presente investigación se busca caracterizar la intención emprendedora en los proyectos universitarios planteando tres indicadores correspondientes al perfil del emprendedor, sector y giro económico, así como aquellos factores que contribuyan a la iniciación del proyecto mediante la aplicación de una encuesta digital cuyos resultados permitan reconocer los elementos de la Teoría de Comportamiento Planificado.

La Teoría de Comportamiento Planificado contempla dos tipos de variables determinantes o explicativas de la intención del comportamiento: la actitud hacia el comportamiento y la norma subjetiva del individuo. Por su parte, la intención de emprendimiento estará influida en primer lugar por la actitud hacia el emprendimiento, es decir, por la predisposición global del individuo hacia la creación de su propio negocio y de las consecuencias asociadas a dicha conducta. De este modo, en la medida en que el sujeto considere que crear una empresa es algo positivo en términos generales, su intención de emprendimiento será mayor. Por otra parte, el propósito de crear un negocio propio estará también influido por la presión social percibida por los individuos respecto a dicha conducta. 
Es decir, la intención de emprendimiento será mayor cuando los sujetos perciben que su entorno social respalda, o al menos no rechaza, dicho comportamiento (Rueda Sampero, Fernández-Laviada, \& Herrero Crespo, 2013, págs. 144-145).

La actitud hacia el emprendimiento esta representada por el perfil del emprendedor que comprende las variables de edad, fuentes de contribución, factores económicos como no económicos. Respecto a la norma subjetiva se materializa en el indicador de sector y giro económico y de los factores que contribuyen en la iniciación del proyecto universitario.

\section{Método}

El diseño de la investigación es no experimental de tipo transversal que consiste en recolectar datos en un solo momento, en un tiempo único, es decir, se pretende describir las características, las propiedades, los elementos constitutivos de un problema de investigación. Con un enfoque cuantitativo, donde se busca analizar la información, a través de la aplicación de un cuestionario.

Los proyectos universitarios comprenden aquellos que se gestan al interior de la materia de creación y desarrollo de empresa en distintos programas académicos educativos que se obtienen de los institutos como son: Instituto de Ciencias Sociales y Administración (ICSA), Instituto de Ingeniería y Tecnología (IIT), Instituto de Ciencias Biomédicas (ICB), Instituto de Arquitectura, Diseño y Arte (IADA) y Ciudad Universitaria (CU, convergen licenciaturas de distintas áreas de especialidad) en la Universidad Autónoma de Ciudad Juárez en Ciudad Juárez, Chihuahua.

\section{Hipótesis}

Hipótesis 1: La actitud de la intención emprendedora está asociada al perfil de emprendedor en los proyectos universitarios en tiempos de COVID-19 independientemente del instituto.

Hipótesis 2: La norma subjetiva de la intención emprendedora esta asociada al sector y giro económico en los proyectos universitarios en tiempos de COVID-19 independientemente del instituto.

\section{Variables}

La herramienta digital que se aplica consiste en un cuestionario que consta de 24 preguntas cuya adaptación de papel a digital se hace al utilizar la aplicación de Forms en la plataforma de Teams, a continuación, se muestra las variables de la encuesta en la Tabla1. 
Tabla 1. Variables de la encuesta

\begin{tabular}{|l|l|l|l|}
\hline Variable & Notación & Medición & $\begin{array}{l}\text { Número de } \\
\text { Pregunta }\end{array}$ \\
\hline Datos Generales & & & \\
\hline Contacto & CON & Correo & 1 \\
\hline Nombre del contacto & NOM & Nombre & 2 \\
\hline Características & MAT & Matrícula & 3 \\
\hline Nombre comercial & PROY & Nombre del proyecto & 4 \\
\hline Asesor & ASE & Asesor & 5 \\
\hline Tipo de proyecto & CAT & Tipo de proyecto & 6 \\
\hline Datos referente al proyecto & & & \\
\hline Sector & SEC & Tipo de sector & 7 \\
\hline Giro & GIR & Tipo de giro & 8 \\
\hline Actividad & ACT & Tipo de actividad económica & 9 \\
\hline Tipo de producto & PRO/SEV & Clasificación de producto & 10 \\
\hline Dimensiones del producto & DIM & Características del producto & 11 \\
\hline Fotos del producto & FOT & Número de Imágenes & 12 \\
\hline Inversión & INVER & Monto de inversión & 13 \\
\hline $\begin{array}{l}\text { Fuentes de contribución a la } \\
\text { identificación del proyecto }\end{array}$ & FUEN & Tipo de fuentes para ideas & 14 \\
\hline Factores Económicos & FE & Número factores económicos & 15 \\
\hline Factores no Económicos & FNE & Número factores no económicos & 16 \\
\hline Documento de apoyo & DOC & Tipo de Herramienta y/o reporte usado & 17 \\
\hline $\begin{array}{l}\text { Constitución Legal } \\
\text { Factores para la iniciación } \\
\text { del proyecto }\end{array}$ & LEG & $\begin{array}{l}\text { Número de proyectos con Constitución } \\
\text { Legal }\end{array}$ & 18 \\
\hline Edad actual & Número de factores & 19 \\
\hline $\begin{array}{l}\text { Edad de los participantes } \\
\text { para ser emprendedor }\end{array}$ & EDAE & Rango de edad actual & 20 \\
\hline Instituto_Campus & Rango de edad para ser emprendedor & 22 \\
\hline Nivel educativo & $\begin{array}{l}\text { Identificación de la ubicación del } \\
\text { proyecto }\end{array}$ & 23 \\
\hline & Grado académico & 24 \\
\hline & NIED & EDA & \\
\hline
\end{tabular}

Fuente: Elaboración propia

\section{Plan de muestreo}

\section{Población}

La población comprende los proyectos universitarios de la UACJ que se definen cada semestre en los diferentes institutos en los grupos de la materia de creación y desarrollo de empresas.

\section{Unidad de la muestra}

La unidad de la muestra son los proyectos universitarios de la materia de creación y desarrollo de empresas, donde se pretende realizar una muestra aleatoria estratificada distinguiendo las características de cada instituto. 
Tamaño de la muestra

La encuesta digital se aplica en el periodo del día 20 de abril al 7 de mayo de 2020 a los estudiantes de los planteles ICSA, IIT, ICB, IADA y CU con una muestra aleatoria estratificada de 83 proyectos en el evento Emprendízate 2020-1 que organiza la Universidad Autónoma de Ciudad Juárez (UACJ).

Procedimiento del muestreo

El procedimiento de selección de la muestra contempla seis etapas considerar los datos de la Tabla 2:

Etapa 1. Identificar de los líderes de cada proyecto universitario

Etapa 2. Distinguir la información de cada proyecto universitario

Etapa 3. Elaborar bases de datos que constituyen el registro de los diferentes proyectos universitarios

Etapa 4. Contactar vía electrónica.

Etapa 5. Depurar y actualizar la base de datos.

Etapa 6. Determinar el número de proyectos universitarios que se puedan con base a las características de la investigación

Tabla 2. Base de datos estimada de proyectos universitarios en UACJ

\begin{tabular}{|c|c|}
\hline Número de proyectos & Tipo de innovación \\
\hline 9 & Radical \\
\hline 9 & Tipo de sistema \\
\hline 32 & Incremental \\
\hline 16 & De nueva generación \\
\hline 14 & Producto \\
\hline
\end{tabular}

Fuente: Elaboración propia

Recolección de datos

Instrumento de medición

El cuestionario consta de 24 preguntas incluye dos apartados, uno con los datos generales del líder del proyecto y otra parte con los datos del proyecto en la que se diferencien tres indicadores: perfil del emprendedor, sector y giro económico como los factores que contribuyen a la etapa de gestación.

El perfil del emprendedor incluye edades, fuentes de contribución a la identificación del negocio, factores económicos y factores no económicos. En el sector y giro económico incorpora el tipo de proyecto (tradicional, tecnológico o social), giro de la empresa (comercio, industria, servicio u otro), actividades económicas según INEGI. Finalmente, en la etapa de gestación se encuentran los factores requeridos para la iniciación del negocio (capacitación, recursos humanos, entidades financieras, trámites u otros). 


\section{Fuentes de información y trabajo de campo}

Para la recolección de la información de los proyectos universitarios es través de una encuesta digital. En la Tabla 3, se presenta las fuentes de información y variables.

Tabla 3. Fuentes de información y variables

\begin{tabular}{|c|c|}
\hline Estructura del Cuestionario & Variables Explicativas \\
\hline \multicolumn{2}{|l|}{ Datos Generales } \\
\hline Dirección de correo electrónico & Contacto \\
\hline Nombre Completo & Nombre del contacto \\
\hline Matricula & Características \\
\hline Nombre del proyecto & Nombre comercial \\
\hline Nombre del asesor & Asesor \\
\hline Tipo de proyecto (categoría) & Tipo de proyecto \\
\hline \multicolumn{2}{|l|}{ Datos referente al proyecto } \\
\hline Sector de la empresa & Sector \\
\hline Giro de la empresa & Giro \\
\hline Actividad principal del proyecto & Actividad \\
\hline Producto/Servicio & Tipo de producto \\
\hline Describa a detalle la función de su producto/servicio & Dimensiones del producto \\
\hline Agregue una(s) imagen(s) de su proyecto. (mínimo 2) & Fotos del producto \\
\hline ¿Cuánto dinero necesita para iniciar o continuar con su proyecto? & Inversión \\
\hline $\begin{array}{l}\text { Señale cuales de las fuentes que se detallan a continuación } \\
\text { contribuyendo a identificar su idea de proyecto }\end{array}$ & $\begin{array}{l}\text { Fuentes de contribución a la } \\
\text { identificación del proyecto }\end{array}$ \\
\hline $\begin{array}{l}\text { Señale en qué medida los siguientes factores económicos pueden } \\
\text { afectar su decisión de iniciar su proyecto. }\end{array}$ & Factores Económicos \\
\hline $\begin{array}{l}\text { Señale en qué medida los siguientes factores no económicos } \\
\text { pueden afectar su decisión de iniciar su proyecto. }\end{array}$ & Factores no Económicos \\
\hline $\begin{array}{l}\text { Indique cuál de los siguientes documentos a elaborado con su } \\
\text { asesor }\end{array}$ & Documento de apoyo \\
\hline Su proyecto está constituido legalmente & Constitución Legal \\
\hline $\begin{array}{l}\text { Evalúe cual es el grado de importancia de los siguientes factores } \\
\text { para abrir su proyecto. }\end{array}$ & $\begin{array}{l}\text { Factores para la iniciación del } \\
\text { proyecto }\end{array}$ \\
\hline Edad actual & Edad actual \\
\hline ¿A qué edad se propuso ser emprendedor? & $\begin{array}{l}\text { Edad de los participantes para ser } \\
\text { emprendedor }\end{array}$ \\
\hline Instituto & Instituto_Campus \\
\hline Grado académico en curso & Nivel educativo \\
\hline
\end{tabular}

Fuente: Elaboración propia

En la Tabla 4 contiene la ficha técnica donde se enlistan las características de la investigación. 
Tabla 4. Ficha técnica de la investigación

\begin{tabular}{|l|l|}
\hline Unidad de análisis & Información \\
\hline Población: & Proyectos universitarios \\
\hline Tamaño de muestra & 83 proyectos \\
\hline Ámbito geográfico: & Universidad Autónoma de Ciudad Juárez \\
\hline Fuentes de información: & $\begin{array}{l}\text { Primaria: Cuestionario } \\
\text { Secundaria: base de datos confidenciales }\end{array}$ \\
\hline Fecha de trabajo de campo: & Del 20 abril al 7 de mayo 2020. \\
\hline Tratamiento de la información: & SPSS versión 26 \\
\hline
\end{tabular}

Fuente: Elaboración propia

Aplicación del instrumento de investigación

El trabajo de campo se realiza en tres etapas: primero, envío de una invitación por correo electrónico; segundo, obtener las respuestas de la encuesta digital y tercero, la revisión y análisis de la información recibida.

Tratamiento y análisis estadístico de datos

Para el tratamiento de los datos se utiliza el software SPSS con la finalidad de elaborar un análisis descriptivo que incluye las respuestas sobre el perfil del emprendedor, el sector y giro del proyecto como tal, así como los factores de la etapa de gestación.

\section{Resultados y discusión}

Respecto a la caracterización de la intención emprendedora de los proyectos universitarios, enseguida se presenta el análisis descriptivo de la actitud de emprendimiento y la norma subjetiva mediante los tres indicadores: perfil del emprendedor, sector y giro económico, etapa de gestación agrupados por instituto.

Para el perfil del emprendedor comprende cuatro variables: edades, fuentes de contribución a la identificación del negocio, los factores económicos y no económicos. En la Gráfica 1 sobre edades de los líderes del proyecto universitario se encuentra la mayoría en el rango de 21 a 25 años independiente del instituto. En relación con las fuentes que contribuyen a la identificación del proyecto destaca la interactuar con las personas e internet como se muestra en la Gráfica 2. 
Gráfica 1. Edades

PERFIL DEL EMPRENDEDOR

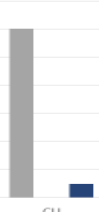

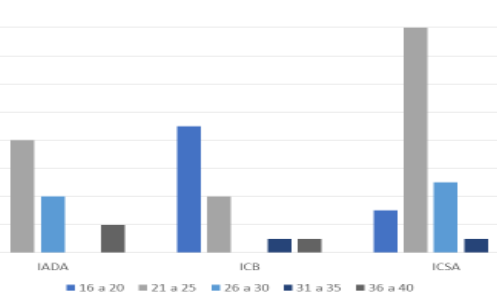

Fuente: Elaboración propia.
Edades

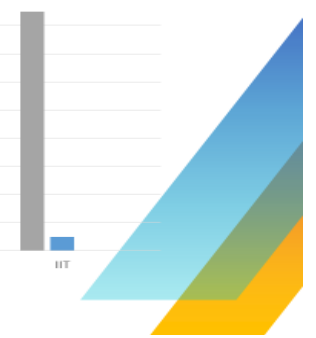

Gráfica 2. Fuentes de contribución a la identificación del negocio.

PERFIL DEL EMPRENDEDOR

\section{Fuentes} Ideas de negocio

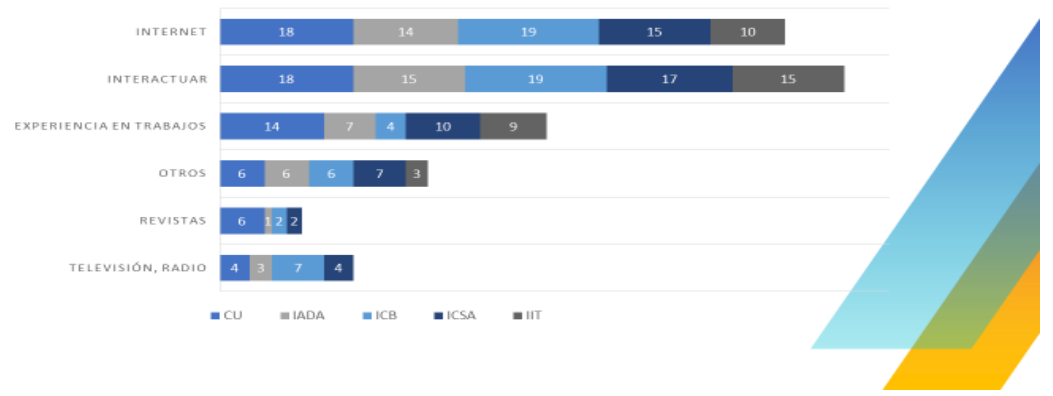

Fuente: Elaboración propia.

En la Gráfica 3, el factor económico que tiene mayor implicación es la disponibilidad de financiamiento. En cambio, los factores no económicos están representado principalmente por la necesidad de contribuir a la sociedad, el desarrollarse como persona y enfrentar continuos desafíos como se muestra en la Gráfica 4. 
Gráfica 3. Factores económicos

\section{PERFIL DEL EMPRENDEDOR}

Factores

Económicos

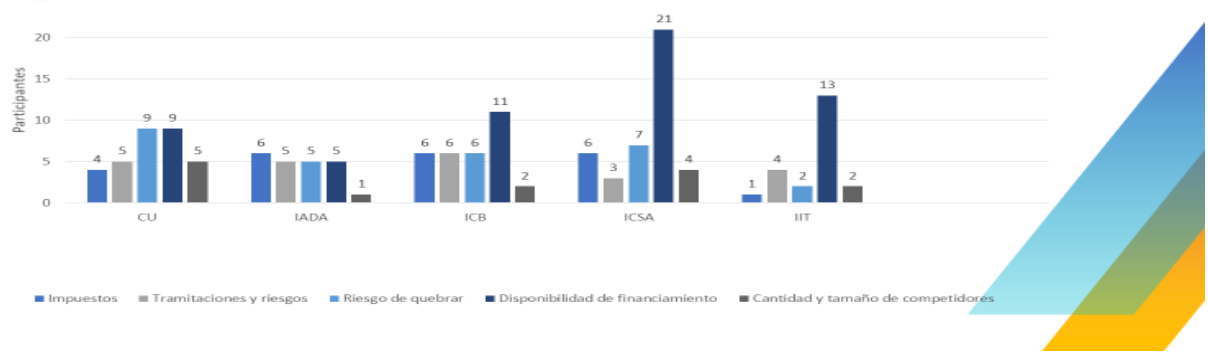

Fuente: Elaboración propia

Gráfica 4. Factores no económicos.

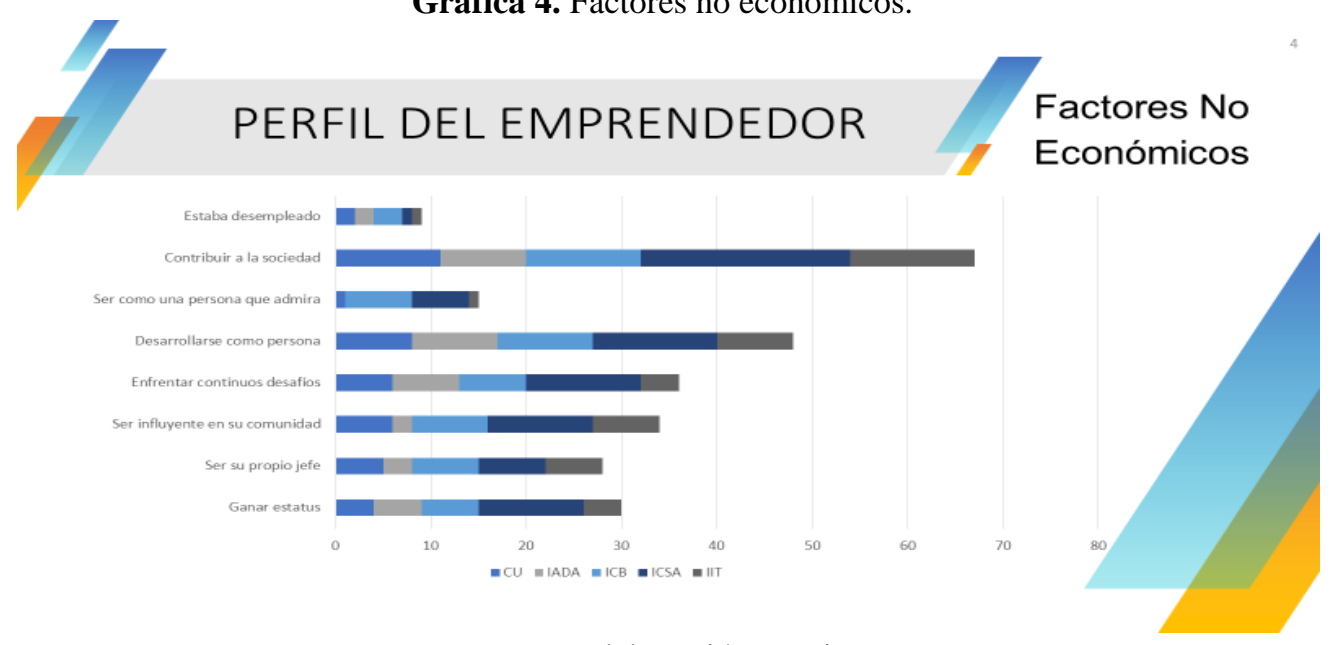

Fuente: Elaboración propia.

Los resultados del perfil del emprendedor representan la actitud del emprendimiento respecto a las creencias de que el comportamiento conduce a ciertos resultados como parte de la intención emprendedora en los proyectos universitarios.

En cuanto a la norma subjetiva de la intención emprendedora se deriva del indicador del sector y giro económico que distinguen tres clasificaciones: una de ellas diferencia a los proyectos tradicional, tecnológico o social en el ámbito académico. Mientras que en la perspectiva empresarial se encuentra la clasificación de comercio, servicio e industrial. Y la tercera clasificación desglosa las actividades económicas según INEGI.

En la Gráfica 5, tipo de proyecto existe una relación directa entre los proyectos tecnológicos con el instituto que se especializa en ingeniería (IIT), por el contrario, los tradicionales existen independiente del instituto. 
Aunque en el giro de la empresa se traduce en servicios y comercio en la Gráfica 6. Respecto a las actividades económicas destacan los servicios de salud y de asistencia social independente del área de especialización de los institutos que representan una correspondencia al contexto actual del COVID19 en la Gráfica 7.

Gráfica 5. Tipo de Proyecto.

\section{SECTOR Y GIRO ECONÓMICO} Tipo de proyecto

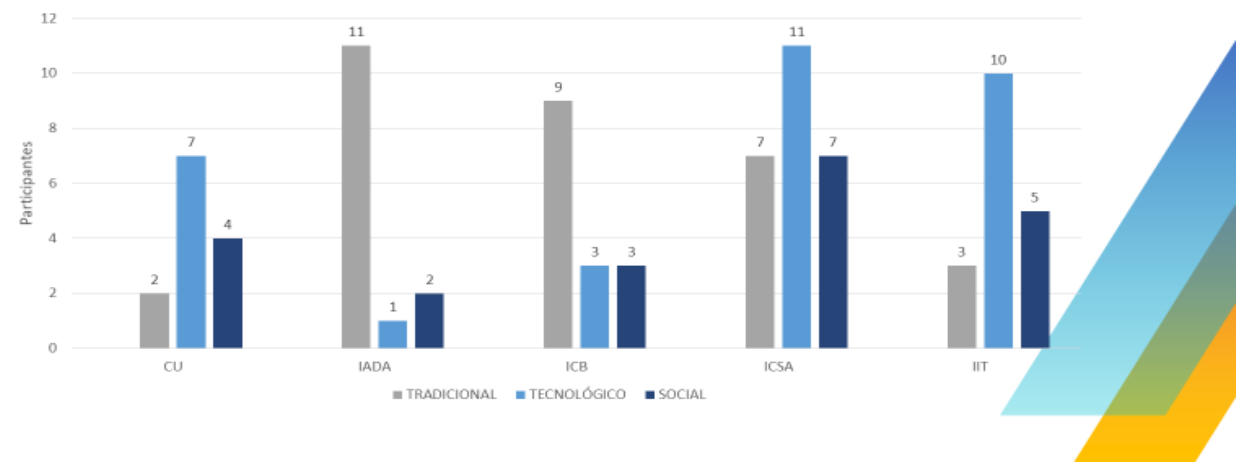

Fuente: Elaboración propia.

Gráfica 6. Giro de la empresa.

\section{SECTOR Y GIRO ECONÓMICO} Giro de la empresa

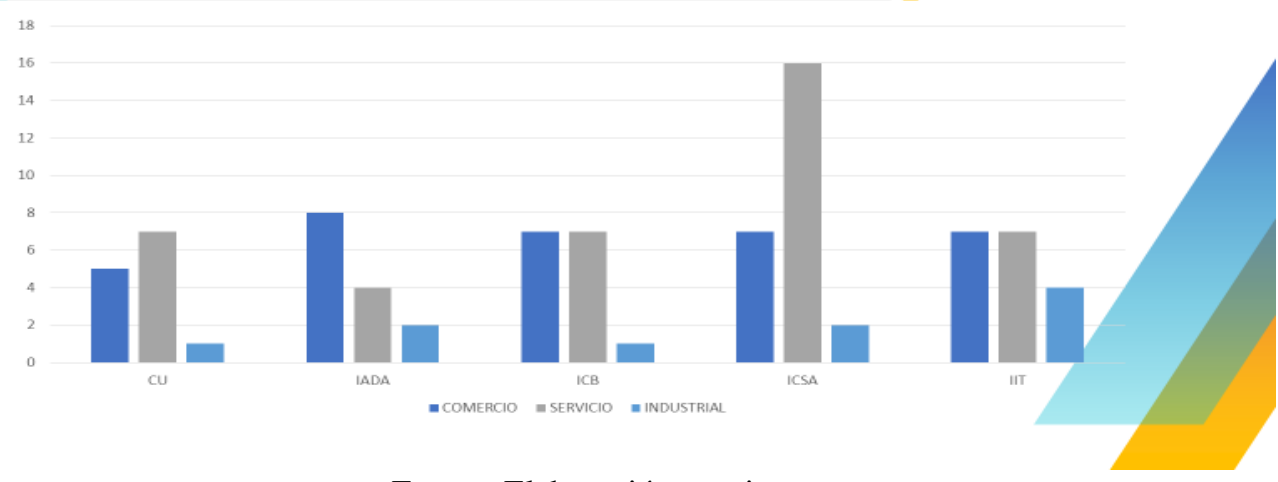

Fuente: Elaboración propia. 


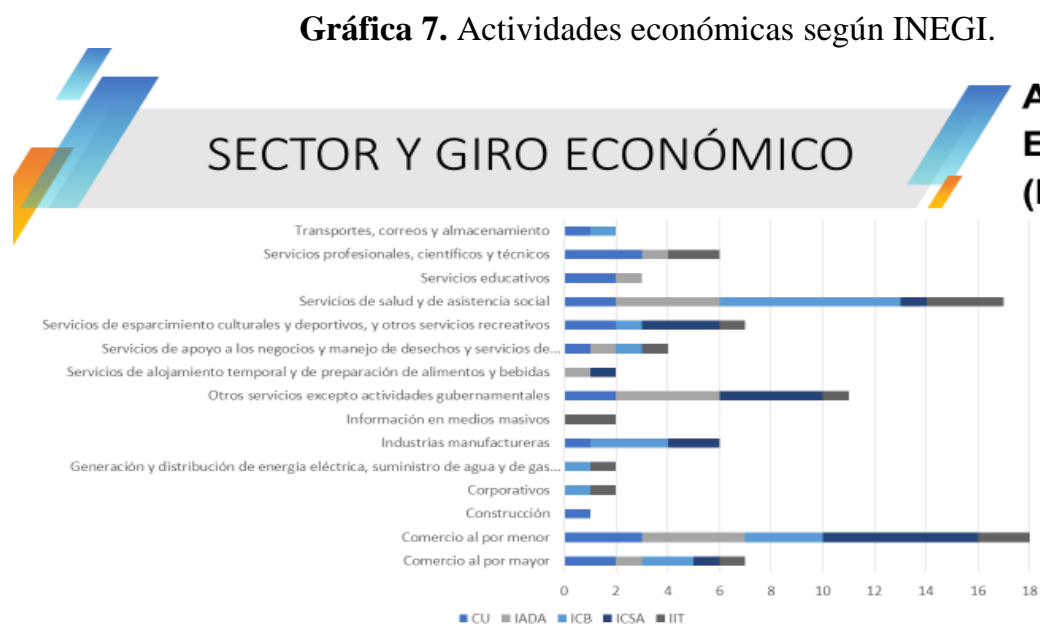

Actividades Económicas (INEGI)

Fuente: Elaboración propia.

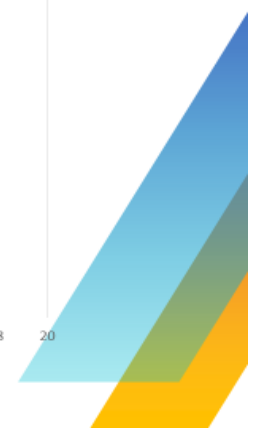

La actitud de emprendimiento y la norma subjetiva de la intención emprendedora puede ser fortalecida con los factores de capacitación y recursos humanos idóneos para iniciar los proyectos universitarios con base a lo obtenido en la Gráfica 8.

Gráfica 8. Factores para iniciación del negocio.

\section{ETAPA DE GESTACIÓN}

Fuente: Elaboración propia.

\section{Conclusiones}

La caracterización de la intención emprendedora en los proyectos universitarios se materializa a través del perfil del emprendedor que representa la actitud del emprendimiento que es resultado de las creencias del individuo y por otro lado la norma subjetiva que se establece con tres tipos de clasificaciones de los proyectos universitarios en cuanto a que otros establecen sus creencias que se atribuyen referencias condicionadas. No obstante, se 
pueden capacitar y contar con los recursos idóneos para iniciar los proyectos universitarios.

Con base a lo anterior se cumple el objetivo de la presente investigación que es distinguir las características de la intención emprendedora en proyectos de universitarios con base a un evento académico en tiempos de COVID-19.

Por otro lado, hipótesis planteadas se validan con los resultados obtenidos donde se puede afirmar que tanto la actitud de emprendimiento y la norma subjetiva están asociadas a los tres indicadores propuestos en los proyectos universitarios en tiempos de COVID-19 independientemente del instituto. Cuyo dato relevante es el giro económico de servicios de salud y de asistencia social como respuesta de las creencias en contribuir con la sociedad. Aunque se requiere tanto de financiamiento como de capacitación y recurso humano, dada la fuente de inspiración es interactuar e internet.

Por último, queda claro que, sin importar el área de especialización, las habilidades necesarias para emprender corresponden a los tiempos de COVID-19 que influye en el planteamiento de los proyectos universitarios.

Es notable que para los estudios de emprendedores universitarios se analicen variables similares a las que se analizaron en esta investigación, ya que si desean tener resultados efectivos es muy importante revisar los elementos claves y fundamentales para realizar un buen emprendimiento.

\section{References:}

1. Borrayo Rodríguez, C. L., Valdez Zepeda, A., \& Delgado Melgarejo, B. (2019). Cultura emprendedora en jóvenes universitarios de Guadalajara, México. Revista de Ciencias Sociales (Ve), vol. XXV, núm. 3, 1-15.

2. Casimiro, W. C. (2019). Competencias de emprendimiento empresarial en estudiantes universitarios. Revista Universidad y Sociedad, 61-69.

3. Echeverri-Sánchez, L., Valencia-Arias, A., Benjumea-Arias, M., \& Barrera-Del Toro, A. (2018). Factores que inciden en la intención emprendedora del estudiantado universitario: Un análisis cualitativo. Revista Electrónica Educare, 1-19.

4. Jolonch, X., \& Ferreira, S. (2016). ¿De qué están hechos nuestros emprendedores? Universidad \& Empresa, vol. 18, núm. 30, 179-189.

5. Krauss Delorme, C., Bonomo Odizzio, A., \& Volfovicz Leon, R. (15 de diciembre de 2018). Modelo predictivo de la intención emprendedora universitaria en Latinoamérica. Journal of Technology Management \& Innovation, 84-93. Obtenido de Scielo: https://scielo.conicyt.cl/scielo.php?script=sci_arttext\&pid=S071827242018000400084\&lang=es 
6. Lanero, A., Vázquez, J.-L., \& Muñoz-Adáñez, A. (10 de enero de 2015). Un modelo social cognitivo de intenciones emprendedoras en estudiantes universitarios. Anales de Psicología, 243-259. Obtenido de Scielo:

http://scielo.isciii.es/scielo.php?script=sci_arttext\&pid=S021297282015000100026\&lang=es

7. Nikulin Chandia, C., Viveros Gunckel, P., Dorochi Fernandois, M., Crespo Márquez, A., \& Lay Bobadilla, P. (2017). Metodología para el análisis de problemas y limitaciones en emprendimientos universitarios. Revista Innovar Journal, 91-105.

8. Ochoa Hernández, M., Azueta Flores, J., \& Rangel Lyne, L. (2015). Características emprendedoras en universitarios próximos a egresar de la Facultad de Comercio y Administración de Tampico. Revista de Psicología y Ciencias del Comportamiento de la Unidad Académica de Ciencias Jurídicas y Sociales, 21-35.

9. Rueda Sampero, I., Fernández-Laviada, A., \& Herrero Crespo, Á. (2013). Aplicación de la teoria de la acción razonada al ámbito emprendedor en un contexto universitario. Investigaciones Regionlaes, 141-158.

10. Sánchez-García, J., \& Hernández-Sánchez, B. (2016). Influencia del Programa Emprendedor Universitario(PREU) para la mejora de la actitud emprendedora. Pampa:Revista Interuniversitaria de Estudios Territoriales, 55-75.

11. Soria-Barreto, K., Zuniga-Jara, S., \& Ruiz-Campo, S. (2016). Educación e Intención Emprendedora en Estudiantes Universitarios: Un Caso de Estudio. Formación universitaria, 25-34.

12. Valencia Arias, A., Montoya Restrepo, I., \& Montoya Restrepo, A. (2016). Intención emprendedora en estudiantes universitarios:Un estudio bibliométrico. Intangible Capital , 881-922. 